\title{
Long-term quality-of-life comparison of total gastrectomy and proximal gastrectomy by Postgastrectomy Syndrome Assessment Scale (PGSAS-45): a nationwide multi-institutional study
}

\author{
Nobuhiro Takiguchi $\cdot$ Masazumi Takahashi $\cdot$ Masami Ikeda $\cdot$ Satoshi Inagawa \\ Shugo Ueda $\cdot$ Takayuki Nobuoka $\cdot$ Manabu Ota $\cdot$ Yoshiaki Iwasaki \\ Nobuyuki Uchida $\cdot$ Yasuhiro Kodera $\cdot$ Koji Nakada
}

Received: 25 December 2013/Accepted: 30 March 2014/Published online: 7 May 2014

(c) The International Gastric Cancer Association and The Japanese Gastric Cancer Association 2014

\begin{abstract}
Background Although proximal gastrectomy (PG) is widely accepted as a function-preserving operation for early upper-third gastric cancer, postoperative disorders, such as reflux or gastric stasis, have often been pointed out. From the perspective of postoperative disorder, the choice of total gastrectomy (TG) or PG for such cancers is still controversial. By using the newly developed Postgastrectomy Syndrome Assessment Scale (PGSAS)-45, the quality of life after TG and PG was compared.

Methods The PGSAS-45 consists of 45 items composed of the SF- 8 and GSRS scales and 22 new items. The main outcomes are measured by seven subscales (SS) covering symptoms, physical and mental component summary (SF8), meals (amount and quality), ability to work, dissatisfaction for daily life, and change in body weight. A total of
\end{abstract}

\footnotetext{
N. Takiguchi $(\bowtie)$

Division of Gastroenterological Surgery, Chiba Cancer Center, 666-2 Nitona-cho, Chuo-ku, Chiba 260-0801, Japan

e-mail: ntaki@chiba-cc.jp

M. Takahashi

Division of Gastroenterological Surgery, Yokohama Municipal

Citizen's Hospital, Yokohama, Japan

M. Ikeda

Department of Surgery, Asama General Hospital, Saku, Japan

S. Inagawa

Department of Surgery, University of Tsukuba, Tsukuba, Japan

S. Ueda

Department of Gastroenterological Surgery, Kitano Hospital,

The Tazuke-Kofukai Medical Research Institute, Osaka, Japan

T. Nobuoka

Department of Surgery I, Sapporo Medical University School

of Medicine, Sapporo, Japan
}

2,368 eligible questionnaires were acquired from 52 institutions. From these, 393 patients with TG and 193 patients with PG were selected and compared.

Results The PG was better than TG in terms of body weight loss (TG $13.8 \%$ vs. PG $10.9 \% ; p=0.003$ ), necessity for additional meals (2.4 vs. $2.0 ; p<0.001)$, diarrhea SS ( 2.3 vs. $2.0 ; p=0.048)$, and dumping SS ( 2.3 vs. $2.0 ; p=0.043)$. There were no differences in the other main outcome measures.

Conclusions Proximal gastrectomy appears to be valuable as a function-preserving procedure for early upperthird gastric cancer.

Keywords Proximal gastrectomy - Total gastrectomy · Postgastrectomy syndrome - Quality of life .

Stomach cancer

M. Ota

Department of Surgery II, School of Medicine, Hamamatsu University, Hamamatsu, Japan

Y. Iwasaki

Division of Surgery, Tokyo Metropolitan Cancer and Infectious

Diseases Center Komagome Hospital, Tokyo, Japan

N. Uchida

Department of Surgery, Haramachi Red-Cross Hospital,

Agatsuma, Gunma, Japan

Y. Kodera

Department of Gastroenterological Surgery, Graduate School of Medicine, Nagoya University, Nagoya, Japan

K. Nakada

Department of Surgery, The Jikei University School

of Medicine, Tokyo, Japan 


\section{Introduction}

Gastric cancer remains the second leading cause of cancer death in the world and is the most frequent malignancy in Japan, South America, and Eastern Europe [1, 2]. Longterm survivors after radical gastrectomy have been increasing as the result of better early detection and improved surgical techniques [3-5]. The better surgical outcome has led to greater interest in the quality of life (QOL) of gastrectomized patients. For prevalence of postgastrectomy disorder, the procedures used in gastrectomy for early gastric cancer are designed as functionpreserving operations or various reconstructions to restore postoperative QOL [6]. Although the postgastrectomy disorders greatly influence the living condition (QOL) of gastrectomized patients, there are limits to evaluation of outpatients because of the difficulty in measuring subjective and physical symptoms. In recent years, questionnaires have been developed to create objective rating systems for QOL [7-11]. The Japan Postgastrectomy Syndrome Working Party was founded in order to investigate symptoms and lifestyle changes among patients who have undergone gastrectomy. This Working Party collaboratively developed a questionnaire to evaluate the symptoms, i.e., living status and QOL, among gastrectomized patients. Using this questionnaire, a nationwide, multiinstitution surveillance study was performed.

The frequency of cancers in the upper third of the stomach and gastroesophageal junction has been increasing in both Western and Asian countries [12-15]. Total gastrectomy (TG) and proximal gastrectomy (PG) are operative options for proximal gastric cancer. In PG, the gastric fundic gland region is kept, and gastric-acid secretion and Castle intrinsic factor are maintained, but patients often suffer from reflux or gastric stasis. The choice of TG or PG has been discussed from the viewpoint of postoperative disorders, especially reflux esophagitis and nutrition. By using the newly developed Postgastrectomy Syndrome Assessment Scale (PGSAS-45), QOL after TG and PG for gastric cancer was compared.

\section{Methods}

\section{Patients}

Fifty-two institutions participated in this study. The PGSAS-45 questionnaire was distributed to 2,922 patients between July 2009 and December 2010. Of these forms, $2,520(86.2 \%)$ were retrieved, of which 152 were deemed ineligible because of patient age $>75$ years $(n=90)$, postoperative period $<1$ year $(n=29)$, co-resection of other organs $(n=8)$, and other factors $(n=25)$. As a result, 2,368 questionnaires $(81 \%)$ were decided as eligible for inclusion in various analyses related to the PGSAS45. Of these, 393 patients who had undergone TG and 193 who had undergone PG were identified and retrieved for the current study (Fig. 1).

\section{Patient eligibility criteria}

Patient eligibility criteria were: (1) pathologically confirmed stage IA or IB gastric cancer; (2) first-time gastrectomy; (3) age $\geq 20$ and $\leq 75$ years; (4) no history of chemotherapy; (5) no known recurrence or distant metastasis; (6) gastrectomy conducted one or more years prior to the enrollment date; (7) performance status (PS) $\leq 1$ on the Eastern Cooperative Oncology Group (ECOG) scale; (8) full capacity to understand and respond to the questionnaire; (9) no history of other diseases or operations that might influence the responses to the questionnaire; (10) no organ failure or mental illness; and (11) provision of written informed consent. Patients with dual malignancy or concomitant resection of other organs (with co-resection equivalent to cholecystectomy being the exception) were excluded.

\section{QOL assessment}

The PGSAS-45 is a newly developed, multidimensional QOL questionnaire (QLQ) based on the Short-Form Health Survey (SF-8) [16] and the Gastrointestinal Symptom Rating Scale (GSRS) [17-20]. The PGSAS-45 questionnaire consists of 45 questions, with eight items from the SF- 8,15 from the GSRS, and 22 clinically important items selected by the Japan Postgastrectomy Syndrome Working Party (Table 1). The PGSAS- 45 questionnaire includes 23 items pertaining to postoperative symptoms (items 9-33), including 15 items from the GSRS and eight newly selected items. In addition, 12 questionnaire items pertaining to dietary intake, work, and level of satisfaction for daily life are included. Dietary intake items include five about the amount of food ingested (items 34-37 and 41) and three about the quality of ingestion (items 38-40). One questionnaire item pertains to work (item 42), while three address the level of satisfaction for daily life (items 42-45). For the 23 symptom items, a seven-grade (1-7) Likert scale is used. A five-grade (1-5) Likert scale is used for all other items except 1, 4, 29, 32, and 34-37. For items $1-8,34,35$ and $38-40$, higher scores indicate better conditions. For items 9-28, 30, 31, 33, and 41-45, higher scores indicate worse conditions. The main outcome measures were refined through consolidation and selection. Twenty-three symptom items were consolidated into seven symptom subscales by factor analysis, as listed in Tables 1 and 2. Assessment data include total symptom score, quality of ingestion subscale, level of satisfaction for daily life, physical component summary (PCS), and mental component 
Fig. 1 Outline of the study

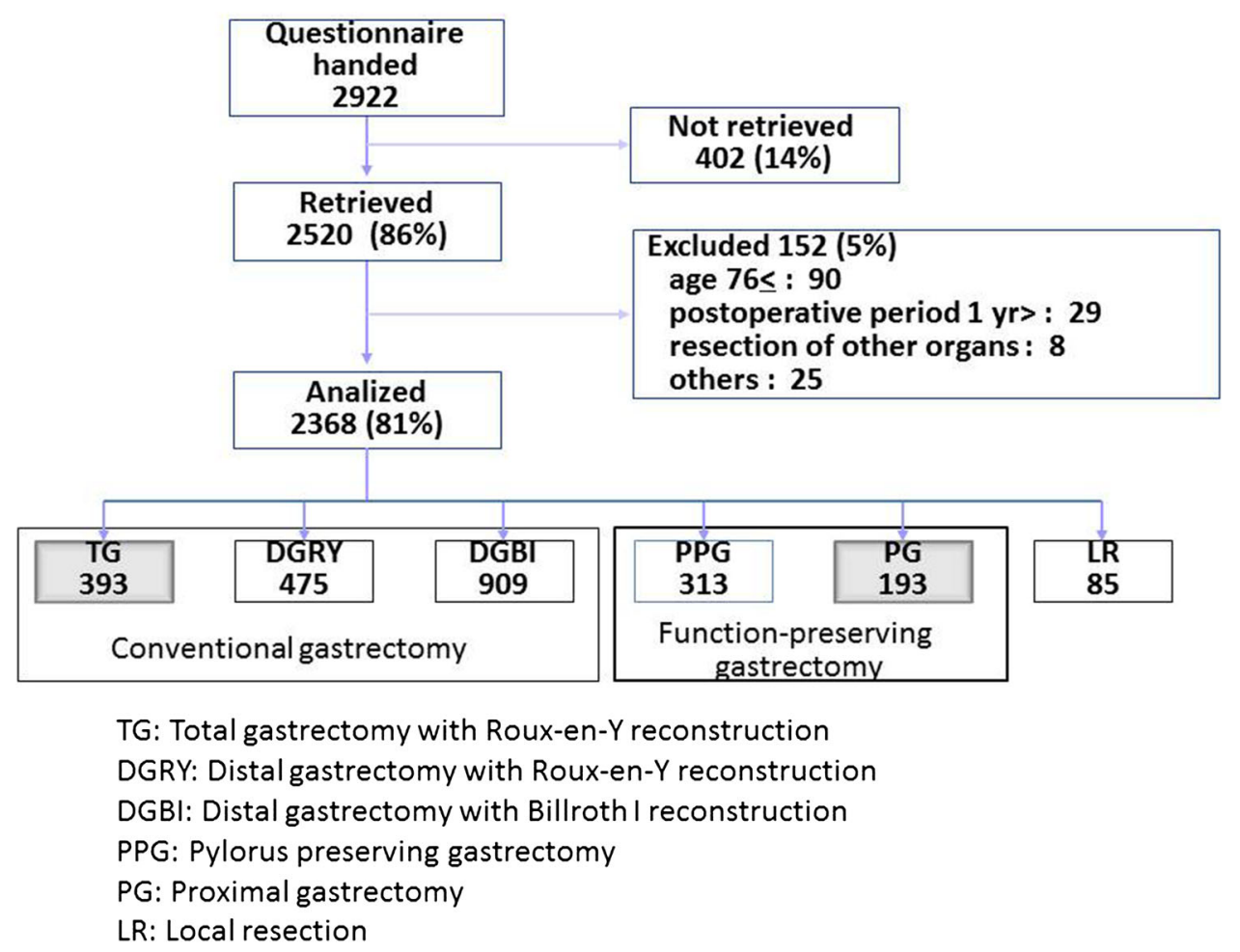

summary (MCS) of the SF-8 as main outcome measures. In addition, the following results were selected as main outcome measures: changes in body weight, amount of food ingested per meal, necessity for additional meals, ability to work, dissatisfaction with symptoms, dissatisfaction at the meal, and dissatisfaction at working. Each subscale score is calculated as the mean of composed items, and the total symptom score is calculated as the mean of seven symptom subscales (Table 2).

Study methods

This study utilized continuous sampling from a central registration system for participant enrollment. The questionnaire was distributed to all eligible patients as they presented to participating clinics. Patients were instructed to return completed forms to the data center. All QOL data from questionnaires were matched with individual patient data collected via case report forms.

This study was registered with the University Hospital Medical Information Network's Clinical Trials Registry (UMIN-CTR; registration number 000002116). It was approved by the ethics committees at all institutions. Written informed consent was obtained from all enrolled patients.

\section{Statistics}

In comparing patient QOLs after TG and PG, statistical methods included the $t$ test and Chi square test. All outcome measures that exhibited significant difference in univariate analysis were further analyzed using multiple regression analysis. $p<0.05$ was considered statistically significant. In the case of $p<0.1$ by univariate analysis, Cohen's $d$ was calculated. In the case of $p<0.1$ in multiple regression analysis, standardization coefficient of regression $(\beta)$, a decision coefficient $\left(R^{2}\right)$, and the $p$ value were calculated and shown in a table. Cohen's $d, \beta$, and $R^{2}$ measure effect sizes. Interpretation of effect sizes were 0.2 $\leq$ small, $0.5 \leq$ medium, and $0.8 \leq$ large in Cohen's $d ; 0.1$ $\leq$ small, $0.3 \leq$ medium, and $0.5 \leq$ large in $\beta$; and $0.02 \leq$ small, $0.13 \leq$ medium, and $0.26 \leq$ large in $R^{2}$.

StatView software for Windows Ver. 5.0 (SAS Institute Inc.) was used for all statistical analyses.

\section{Results}

Patient characteristics

Background data of both groups of patients are shown in Table 3. Reconstruction procedures were not regulated by the protocol, and depended on the principle of the institution or discretion of each surgeon. Consequently, whereas all patients treated by TG (393 patients) underwent Roux en $Y$ reconstruction, the reconstruction after PG (193 patients) was varied and consisted of gastro-esophagostomy (115 patients), jejunal interposition (34 patients), and jejunal pouch interposition (44 patients). 


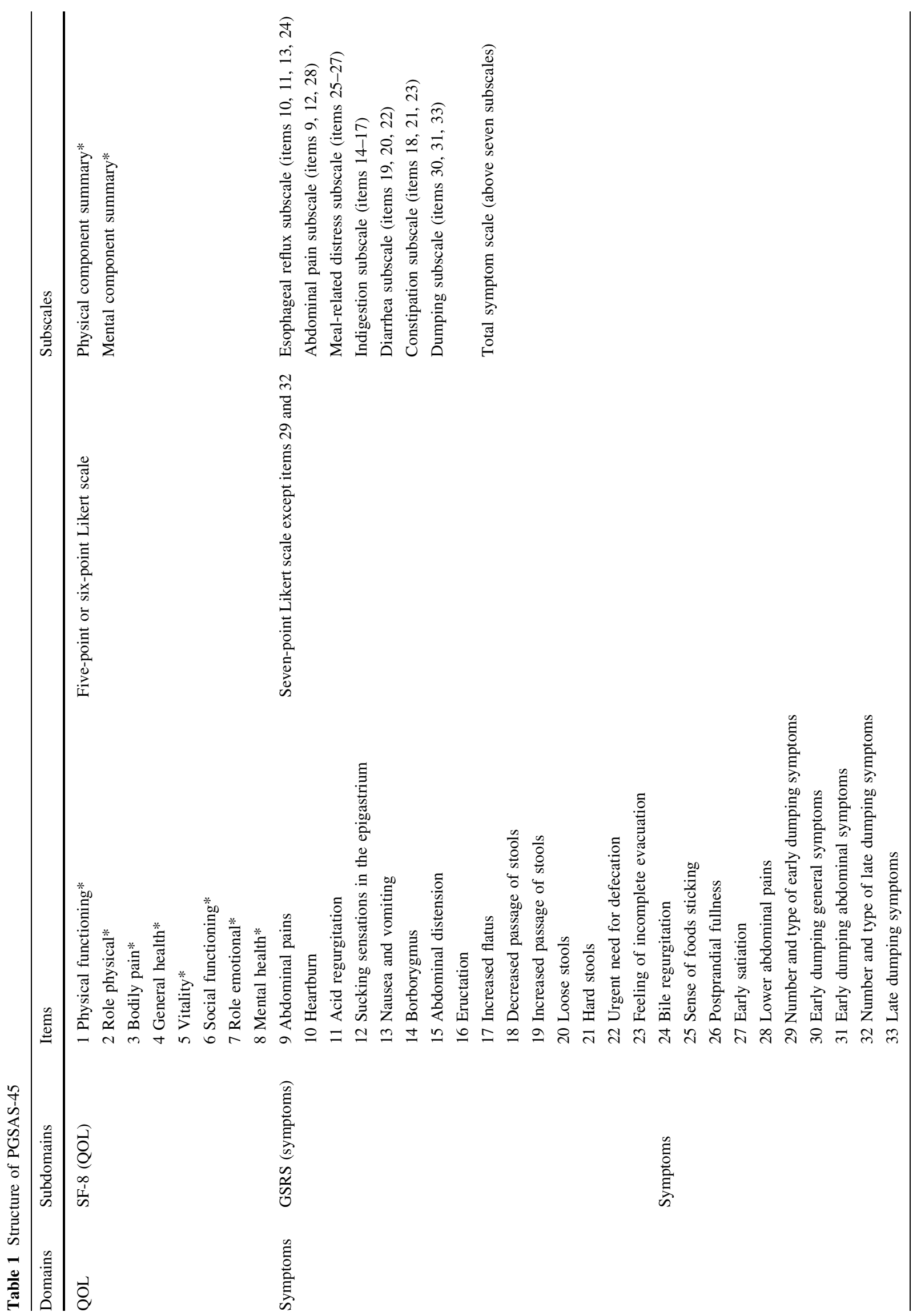




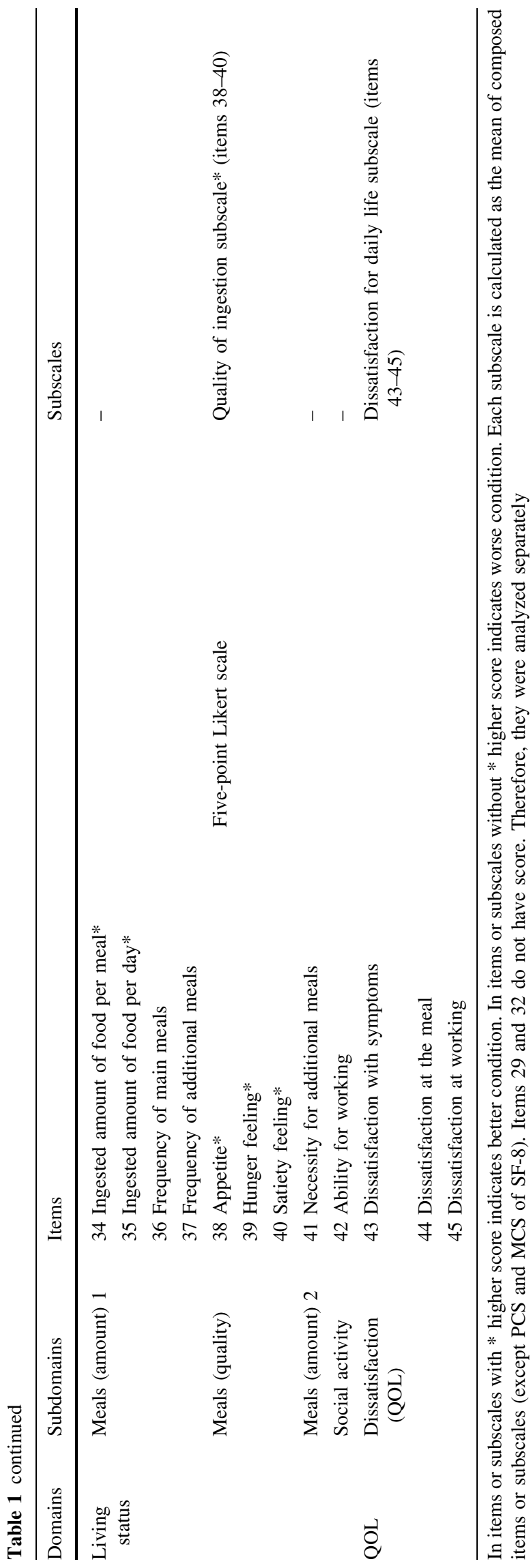

Table 2 Domains and main outcome measures

\begin{tabular}{|c|c|c|}
\hline \multicolumn{2}{|c|}{ Domains/subdomains } & \multirow{2}{*}{$\begin{array}{l}\text { Main outcome measures } \\
\text { Seven symptom subscales }\end{array}$} \\
\hline Symptoms & Subscales & \\
\hline & & $\begin{array}{l}\text { Esophageal reflux }(10,11,13,24), \\
\text { abdominal pain }(9,12,28), \text { meal- } \\
\text { related distress }(25-27), \text { indigestion } \\
(14-17), \text { diarrhea }(19,20,22), \\
\text { constipation }(18,21,23), \text { dumping } \\
(30,31,33)\end{array}$ \\
\hline & Total & Total symptom score \\
\hline \multirow{5}{*}{$\begin{array}{l}\text { Living } \\
\text { status }\end{array}$} & Body weight & Change in body weight $(\%)^{*}$ \\
\hline & $\begin{array}{l}\text { Meals } \\
\quad \text { (amount) }\end{array}$ & $\begin{array}{l}\text { Ingested amount of food per meal* } \\
\text { (34) }\end{array}$ \\
\hline & & Necessity for additional meals (41) \\
\hline & $\begin{array}{l}\text { Meals } \\
\quad \text { (quality) }\end{array}$ & Quality of ingestion subscale* (38-40) \\
\hline & Work & Ability for working (42) \\
\hline \multirow[t]{4}{*}{ QOL } & Dissatisfaction & $\begin{array}{l}\text { Dissatisfaction with symptoms (43), at } \\
\text { the meal (44), at working (45) }\end{array}$ \\
\hline & & $\begin{array}{l}\text { Dissatisfaction for daily life subscale } \\
(43-45)\end{array}$ \\
\hline & SF-8 & Physical component summary* (1-5) \\
\hline & & Mental component summary* (4-8) \\
\hline
\end{tabular}

Main outcome measures that are italicized are composed of more than two items. In items or subscales with *, higher score indicates better condition; in items or subscales without $*$, higher score indicates worse condition. Each subscale is calculated as the mean of composed items or subscales

In the PG group, the mean postoperative period was significantly longer (TG $35.0 \pm 24.6$ months vs. PG $40.5 \pm 28.1$ months; $p=0.0163$ ), and the rates of celiac and pyloric branch preservation were significantly higher, while the rates of laparoscopic approaches, D2 lymph node dissection, and combined resections were significantly lower than in the TG group.

QOL assessments

The results of the main outcome measures by univariate analysis are shown in Table 4 . The body weight loss (TG $13.8 \%$ vs. PG $10.9 \% ; p=0.0001$; Cohen's $d=0.35$ ), diarrhea subscale (TG 2.3 vs. PG 2.0; $p=0.0016$; Cohen's $d=0.29$ ), and dumping subscale (TG 2.3 vs. PG $2.0 ; p=0.0118$; Cohen's $d=0.24$ ) in the PG group were significantly lower than those in the TG group.

The necessity for additional meals was significantly lower in the PG group than in the TG group (TG 2.4 vs. PG 2.0; $p<0.001$; Cohen's $d=0.40$ ), which indicates a better status in the PG group. However, the constipation subscale value of the PG group was significantly higher than that of the TG group (TG 2.1 vs. PG $2.3 ; p=0.0145$; Cohen's $d=0.21$ ), and the quality of ingestion subscale value of the PG group was significantly lower than that of 
Table 3 Patient background and operative features

$T G$ Roux en Y reconstruction $(n=393)$; $P G$ Gastro-

esophagostomy $(n=115)$, Jejunal interposition $(n=34)$, Jejunal pouch interposition $(n=44)$

Table 4 Main outcome measures by univariate analysis

Integrated subscales are italicized in the table

For outcome measures with * higher score indicates better condition; for outcome measures without $*$ higher score indicates worse condition

\begin{tabular}{llll}
\hline Type of gastrectomy & TG & PG & $p$ value \\
& Mean (SD) & Mean (SD) & \\
\hline Number of patients & 393 & 193 & 0.0163 \\
Postoperative period (months) & $35.0(24.6)$ & $40.5(28.1)$ & $>0.1$ \\
Age & $63.4(9.2)$ & $63.7(7.7)$ & $>0.1$ \\
Sex (male/female) & $276 / 113$ & $139 / 53$ & $>0.1$ \\
BMI (preoperative) & $23.0(3.3)$ & $23.1(3.0)$ & 0.0364 \\
Operation background & & & $<0.0001$ \\
Approach (laparoscopic/open) & $97 / 293$ & $33 / 159$ & $<0.0001$ \\
Celiac branch of vagus (preserved/divided) & $12 / 371$ & $83 / 105$ & $<0.0001$ \\
Pyloric branch of vagus (preserved/divided) & $4 / 379$ & $120 / 62$ & \\
Extent of lymph node dissection & & & \\
D2 & 164 & 7 & \\
D1b & 192 & 93 & \\
D1a & 28 & 72 & \\
D1 & 4 & 7 & \\
D1> & 0 & 6 & \\
None & 0 & 0 & \\
Combined resection & & 14 & \\
Cholecystectomy & 83 & 162 & \\
Splenectomy & 52 & & \\
Others & 246 & & \\
None & & & \\
\hline & & & \\
\end{tabular}

\begin{tabular}{|c|c|c|c|c|c|c|}
\hline \multirow[t]{2}{*}{ Measure } & \multicolumn{2}{|l|}{ TG } & \multicolumn{2}{|l|}{ PG } & \multirow[t]{2}{*}{ Cohen's $d$} & \multirow[t]{2}{*}{$p$ value } \\
\hline & Mean & SD & Mean & SD & & \\
\hline Change in body weight* & $-13.80 \%$ & $7.90 \%$ & $-10.90 \%$ & $8.20 \%$ & 0.35 & 0.0001 \\
\hline Esophageal reflux subscale & 2.0 & 1.0 & 2.0 & 1.0 & & $>0.1$ \\
\hline Abdominal pain subscale & 1.8 & 0.8 & 1.7 & 0.7 & & $>0.1$ \\
\hline Meal-related distress subscale & 2.6 & 1.1 & 2.6 & 1.1 & & $>0.1$ \\
\hline Indigestion subscale & 2.3 & 0.9 & 2.2 & 0.8 & & $>0.1$ \\
\hline Diarrhea subscale & 2.3 & 1.2 & 2.0 & 1.0 & 0.29 & 0.0016 \\
\hline Constipation subscale & 2.1 & 0.9 & 2.3 & 1.1 & 0.21 & 0.0145 \\
\hline Dumping subscale & 2.3 & 1.1 & 2.0 & 1.0 & 0.24 & 0.0118 \\
\hline Total symptom score & 2.2 & 0.7 & 2.1 & 0.7 & & $>0.1$ \\
\hline Ingested amount of food per meal* & 6.4 & 1.9 & 6.5 & 1.9 & & $>0.1$ \\
\hline Necessity for additional meals & 2.4 & 0.8 & 2.0 & 0.8 & 0.40 & $<0.0001$ \\
\hline Quality of ingestion subscale* & 3.8 & 0.9 & 3.6 & 1.0 & 0.20 & 0.0281 \\
\hline Ability for working & 2.0 & 0.9 & 2.0 & 0.9 & & $>0.1$ \\
\hline Dissatisfaction with symptoms & 2.1 & 1.0 & 2.0 & 0.9 & & $>0.1$ \\
\hline Dissatisfaction at the meal & 2.8 & 1.1 & 2.7 & 1.1 & & $>0.1$ \\
\hline Dissatisfaction at working & 2.1 & 1.1 & 2.0 & 1.1 & & $>0.1$ \\
\hline Dissatisfaction for daily life subscale & 2.3 & 0.9 & 2.2 & 0.9 & & $>0.1$ \\
\hline Physical component summary* & 49.6 & 5.6 & 49.5 & 6.1 & & $>0.1$ \\
\hline Mental component summary* & 49.2 & 6.0 & 49.0 & 6.0 & & $>0.1$ \\
\hline
\end{tabular}


the TG group (TG 3.8 vs. PG 3.6; $p=0.0281$; Cohen's $d=$ 0.20 ), both of which indicate worse status of the PG group.

The physical and mental component summaries were not different in the two groups.

To eliminate confounding factors, multiple regression analysis was performed by adding postoperative period, age, sex, surgical approach, and celiac branch of vagal nerve preservation as explanatory variables (Table 5). Although the effect size of the advantages in PG over TG is relatively small, comparing the type of gastrectomy, the PG group was better than the TG group in body weight loss $(\beta=0.148 ; p=0.003)$, diarrhea $(\beta=0.097 ; p=0.048)$, dumping $(\beta=0.106 ; p=0.043)$, and necessity for additional meals $(\beta=0.192 ; p<0.001)$. Constipation and quality of ingestion, which were worse in the PG group by univariate analysis, showed no difference by multivariate analysis.

Multiple regression analysis revealed that the postoperative period influenced the extent of body weight loss $(\beta=0.097 ; p=0.030)$, diarrhea $(\beta=-0.076 ; p=0.078)$, and quality of ingestion $(\beta=0.092 ; p=0.0365)$. This means that as the postoperative period lengthens, body weight loss and diarrhea improve.

The age influenced the constipation subscale $(\beta=0.147$; $p=0.001)$, dumping $(\beta=-0.114 ; p=0.010)$, and the quality of ingestion $(\beta=-0.126 ; p=0.034)$. At older ages, although dumping decreased, constipation increased.

Diarrhea was often found in men $(\beta=0.137$; $p=0.001$ ), and surgical approach and celiac branch preservation had little influence on any of the main outcome measures by multiple regression analysis.

\section{Discussion}

Optimal evaluation methods for postgastrectomy disorders are important for selecting and improving the operative procedures and maintaining the high QOL for gastric cancer patients [21-23]. The Japan Postgastrectomy Syndrome Working Party developed a questionnaire to evaluate general features; i.e., symptoms, living status, and QOL, among gastrectomized patients. Using this questionnaire, a nationwide, multi-institution surveillance study was performed. This was the first nationwide survey of its type and involved 52 medical institutions throughout Japan. The necessary QOL data were collected from 2,520 patients, and the final sample size, following exclusion and participant selection, was sufficient for statistical validity of this type of study.

In recent years, a tendency to increasing numbers of proximal gastric cancers has been reported, and early detection and potentially curative operations by PG for upper-third gastric cancers have been increasing [24, 25].

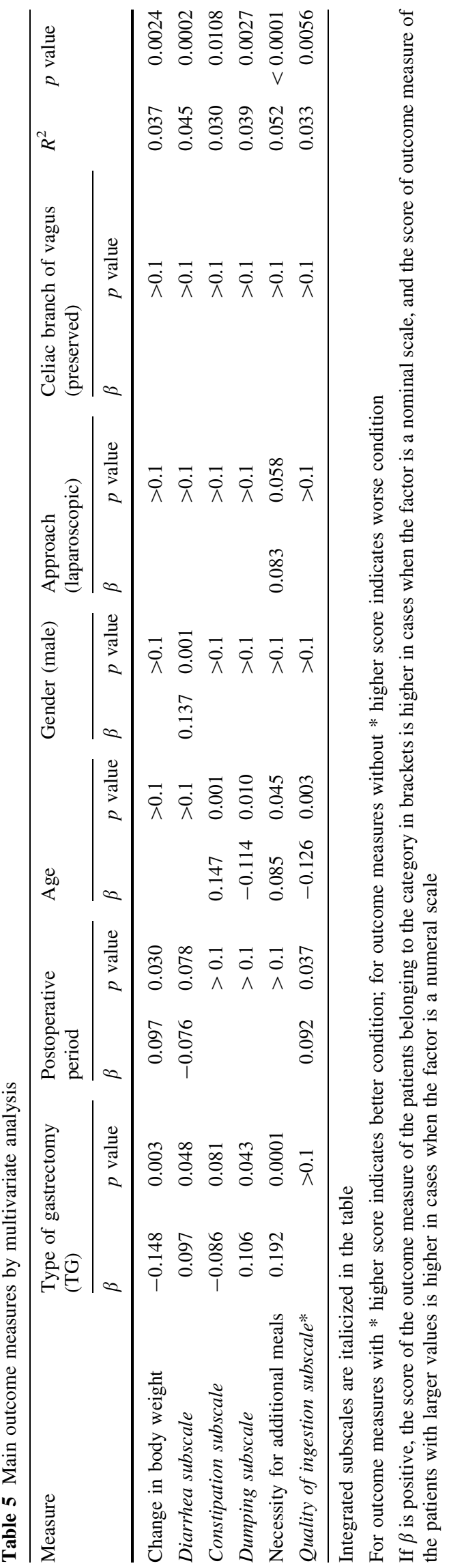


In this study, the effect of tumor progression was removed by constraining patient selection to those with pathologic Stage IA/IB disease, and it is thought that accurate QOL comparison between operative procedures is possible under these circumstances. Although QOL scores usually depend on the time after surgery, Kobayashi et al. [11] reported that the QOL after gastrectomy was impaired during a few months after surgery, but more or less stabilized at around 6 months after surgery. This is the reason that, in this nationwide survey, we chose to evaluate patients who had lived for 12 months or more after surgery. In addition, we used multiple regression analysis with time relapse after surgery as one of variables so as to adjust this problem.

Whereas the reconstruction for TG was only by the Roux-en-Y method, the reconstructions of PG could be by esophagogastrostomy, jejunal interposition, and jejunal pouch interposition [6]. Because the best reconstruction for PG has not yet been established, various procedures are performed. However, as the gastric fundic gland region is preserved in PG, gastric-acid secretion and production of Castle intrinsic factor and ghrelin, a gut hormone known increase to appetite, are maintained [26, 27].

In the PG group, the rates of celiac and pyloric branch vagal nerve preservation were significantly higher, and the rates of laparoscopic approaches, D2 lymph node dissection, and combined resection were significantly lower than in the TG group. Standard TG is composed of more D1b dissection and sacrifice of the vagal nerve, often with combined resection, such as of the spleen and gallbladder $[6,28]$. On the other hand, PG, which is a function-preserving operation, usually consists of less than D1b dissection and preservation of the vagal nerve [6]. The differences in the surgical background are caused by the procedure itself. Therefore, there seems to be no problem in comparing the QOL scores of these two groups.

From the results of the main outcome measures by univariate and multivariate analysis, body weight loss, diarrhea, dumping, and necessity for additional meals were significantly lower in the PG than in the TG group. Although esophageal reflux is common after PG [29, 30], various reconstruction methods have recently been described that reduce this problem [31, 32]. In this study, there was no difference in the esophageal reflux subscale values between the groups. This result suggests that PG is not necessarily disadvantageous with regard to reflux.

As three types of reconstruction with various modifications were performed with PG reconstruction, it is necessary to compare the three procedures in future studies. Dumping symptoms, such as early dumping with systemic symptoms, early dumping with abdominal symptoms, and late dumping, were examined in detail. Late dumping was significantly less common in the PG than in the TG group.
Also, a tendency toward less early dumping with abdominal symptoms was seen in the PG group (data not shown). As a result, PG performed well on the dumping subscale. Although PG reflected the storage capacity and pyloruspreserving function, in TG, solid food is passed rapidly to the jejunum because of no storage ability [33].

Although the constipation subscale results and quality of ingestion subscale values were worse with PG than with TG by univariate analysis, multivariable regression analysis revealed that there were no statistical differences in these subscales as the result of the type of gastrectomy. Body weight loss and quality of ingestion subscale improved if the postoperative period was long. This means that gastrectomized patients adapt in some ways to the anatomic changes over time, even after more than 1 year following gastrectomy.

Multivariable regression analysis showed that dumping decreased and constipation increased with advancing age. This result may reflect the known intestinal peristaltic decrease in older patients [34-37].

By multivariable regression analysis, men were more likely to have diarrhea than women. This may be a consequence of the fact that the intestinal transit time is longer in women than in men at equivalent ages [37-39]. As for the effect of the surgical approaches and celiac branch preservation, no differences were found by multivariable regression analysis.

There were no statistical differences between the groups with regard to ability to work, dissatisfaction with symptoms, dissatisfaction at working, dissatisfaction for daily life subscale, PCS, or MCS. It is suggested that daily life is largely unchanged and that statistically different postgastrectomy disorders do not have a major effect on adaptation.

In conclusion, although the effect size of the advantages of PG over TG is relatively small, our results indicate that PG is useful as a function-preserving procedure for early upper-gastric cancer. Although this study is limited in that it is retrospective and examines a single time point, it suggests the value of PG, use of which should be encouraged. To confirm this conclusion, a randomized study to determine the most desirable reconstruction for $\mathrm{PG}$ to achieve a good long-term QOL will have to be conducted using the PGSAS-45 questionnaire and successive endoscopic examinations.

Acknowledgments This study was supported by a grant from Jikei University and the Japanese Society for Gastro-Surgical Pathophysiology. The authors thank all physicians who participated in this study and the patients whose cooperation made this study possible.

Conflict of interest The authors declare no conflicts of interest with regard to this manuscript. 


\section{References}

1. Ferlay J, Shin HR, Bray F, Forman D, Mathers C, Parkin DM. Estimates of worldwide burden of cancer in 2008: GLOBOCAN 2008. Int J Cancer. 2010;127:2893-917.

2. Jemal A, Bray F, Center MM, Ferlay J, Ward E, Forman D. Global cancer statistics. CA Cancer J Clin. 2011;61:69-90.

3. Tsujimoto H, Sugasawa H, Ono S, Ichikura T, Yamamoto J, Hase $\mathrm{K}$. Has the accuracy of preoperative diagnosis improved in cases of early-stage gastric cancer? World J Surg. 2010;34:1840-6.

4. Kunisaki C, Ishino J, Nakajima S, et al. Outcomes of mass screening for gastric carcinoma. Ann Surg Oncol. 2006;13:221-8.

5. Kim YW, Yoon HM, Yun YH, Nam BH, Eom BW, Baik YH, et al. Long-term outcomes of laparoscopy-assisted distal gastrectomy for early gastric cancer: result of a randomized controlled trial (COACT 0301). Surg Endosc. 2013;27:4267-76.

6. Japanese Gastric Cancer Association. Japanese gastric cancer treatment guidelines 2010 (ver. 3). Gastric Cancer. 2011;14:113-23.

7. Vickery CW, Blazeby JM, Conroy T, Johnson CD, Alderson D. Development of an EORTC module to improve quality of life assessment in patients with gastric cancer. $\mathrm{Br} \mathrm{J}$ Surg. 2000;87:362-73.

8. Blazeby JM, Conroy T, Bottomley A, European Organisation for Research and Treatment of Cancer Gastrointestinal and Quality of Life Groups, et al. Clinical and psychometric validation of a questionnaire module, the EORTC QLQ-STO 22, to assess quality of life in patients with gastric cancer. Eur $\mathrm{J}$ Cancer. 2004;40:2260-8.

9. Kaptein AA, Morita S, Sakamoto J. Quality of life in gastric cancer. World J Gastroenterol. 2005;11:3189-96.

10. Lagergren P, Fayers P, Conroy T, Stein HJ, Sezer O, Hardwick R, et al. Clinical and psychometric validation of a questionnaire module, the EORTC QLQ-OG25, to assess health-related quality of life in patients with cancer of the oesophagus, the oesophagogastric junction and the stomach. Eur J Cancer. 2007;43:2066-73.

11. Kobayashi D, Kodera Y, Fujiwara M, Koike M, Nakayama G, Nakao A. Assessment of quality of life after gastrectomy using EORTC QLQ-C30 and STO22. World J Surg. 2011;35:357-64.

12. Wu H, Rusiecki JA, Zhu K, Potter J, Devesa SS. Stomach carcinoma incidence patterns in the United States by histologic type and anatomic site. Cancer Epidemiol Biomark Prev. 2009;18:1945-52.

13. Steevens J, Botterweck AA, Dirx MJ, van den Brandt PA, Schouten LJ. Trends in incidence of oesophageal and stomach cancer subtypes in Europe. Eur J Gastroenterol Hepatol. 2010;22:669-78.

14. Kusano C, Gotoda T, Khor CJ, Katai H, Kato H, Taniguchi H, Shimoda T. Changing trends in the proportion of adenocarcinoma of the esophagogastric junction in a large tertiary referral center in Japan. J Gastroenterol Hepatol. 2008;23:1662-5.

15. Dassen AE, Lemmens VE, van de Poll-Franse LV, Creemers GJ, Brenninkmeijer SJ, Lips DJ, et al. Trends in incidence, treatment and survival of gastric adenocarcinoma between 1990 and 2007: a population-based study in the Netherlands. Eur $\mathrm{J}$ Cancer. 2010;46:1101-10.

16. Turner-Bowker DM, Bayliss MS, Ware JE Jr, Kosinski M. Usefulness of the SF-8 Health Survey for comparing the impact of migraine and other conditions. Qual Life Res. 2003;12:1003-12.

17. Svedlund J, Sjodin I, Dotevall G. GSRS—a clinical rating scale for gastrointestinal symptoms in patients with irritable bowel syndrome and peptic ulcer disease. Dig Dis Sci. 1988;33:129-34.

18. Wiklund IK, Junghard O, Grace E, Talley NJ, Kamm M, Veldhuyzen van Zanten S, et al. Quality of life in reflux and dyspepsia patients. Psychometric documentation of a new disease- specific questionnaire (QOLRAD). Eur J Surg Suppl. 1998;583:41-9.

19. Hongo M, Fukuhara S, Green J. QOL in digestive region: QOL evaluation by Japanese edition GSRS. Diagn Treat. 1999;87:731-6.

20. Kulich KR, Madisch A, Pacini F, Piqué JM, Regula J, Van Rensburg CJ, et al. Reliability and validity of the gastrointestinal symptom rating scale (GSRS) and quality of life in reflux and dyspepsia (QOLRAD) questionnaire in dyspepsia: a six-country study. Health Qual Life Outcomes. 2008;31(6):12.

21. Katai H. Function-preserving surgery for gastric cancer. Int J Clin Oncol. 2006;11:357-66.

22. Lehnert $\mathrm{T}$, Buhl $\mathrm{K}$. Techniques of reconstruction after total gastrectomy for cancer. Br J Surg. 2004;91:528-39.

23. Hiki N, Nunobe S, Kubota T, Jiang X. Function-preserving gastrectomy for early gastric cancer. Ann Surg Oncol. 2013;20:2683-92.

24. Wen L, Chen XZ, Wu B, et al. Total vs. proximal gastrectomy for proximal gastric cancer: a systematic review and meta-analysis. Hepatogastroenterology. 2012;59:633-40.

25. Ahn SH, Lee JH, Park Do J, Kim HH. Comparative study of clinical outcomes between laparoscopy-assisted proximal gastrectomy (LAPG) and laparoscopy-assisted total gastrectomy (LATG) for proximal gastric cancer. Gastric Cancer. 2013;16:282-9.

26. Kojima M, Hosoda H, Date Y, Nakazato M, Matsuo H, Kangawa $\mathrm{K}$. Ghrelin is a growth-hormone-releasing acylated peptide from stomach. Nature. 1999;402:656-60.

27. Takiguchi S, Hiura Y, Miyazaki Y, Takata A, Murakami K, Doki Y. Clinical trial of ghrelin synthesis administration for upper GI surgery. Methods Enzymol. 2012;514:409-31.

28. Ikeguchi M, Kaibara N. Lymph node metastasis at the splenic hilum in proximal gastric cancer. Am Surg. 2004;70:645-8.

29. An JY, Youn HG, Choi MG, Noh JH, Sohn TS, Kim S. The difficult choice between total and proximal gastrectomy in proximal early gastric cancer. Am J Surg. 2008;196:587-91.

30. Yoo CH, Sohn BH, Han WK, Pae WK. Long-term results of proximal and total gastrectomy for adenocarcinoma of the upper third of the stomach. Cancer Res Treat. 2004;36:50-5.

31. Nozaki I, Hato S, Kobatake T, Ohta K, Kubo Y, Kurita A. Longterm outcome after proximal gastrectomy with jejunal interposition for gastric cancer compared with total gastrectomy. World J Surg. 2013;37:558-64.

32. Tokunaga M, Hiki N, Ohyama S, Nunobe S, Miki A, Fukunaga T, et al. Effects of reconstruction methods on a patient's quality of life after a proximal gastrectomy: subjective symptoms evaluation using questionnaire survey. Langenbecks Arch Surg. 2009;394:637-41.

33. Tack J, Arts J, Caenepeel P, De Wulf D, Bisschops R. Pathophysiology, diagnosis and management of postoperative dumping syndrome. Nat Rev Gastroenterol Hepatol. 2009;6:583-90.

34. Towers AL, Burgio KL, Locher JL, Merkel IS, Safaeian M, Wald A. Constipation in the elderly: influence of dietary, psychological, and physiological factors. J Am Geriatr Soc. 1994;42:701-6.

35. Pilotto A, Franceschi M, Vitale D, Zaninelli A, Di Mario F, Seripa D, Rengo F, FIRI (Fondazione Italiana Ricerca sull'Invecchiamento), SOFIA Project Investigators. The prevalence of diarrhea and its association with drug use in elderly outpatients: a multicenter study. Am J Gastroenterol. 2008;103:2816-23.

36. Aoki T, Yamaji I, Hisamoto T, Sato M, Matsuda T. Irregular bowel movement in gastrectomized subjects: bowel habits, stool characteristics, fecal flora, and metabolites. Gastric Cancer. 2012;15:396-404.

37. Harari D, Gurwitz JH, Avorn J, Bohn R, Minaker KL. Bowel habit in relation to age and gender: findings from the National 
Health Interview Survey and clinical implications. Arch Intern Med. 1996;156:315-20.

38. Probert CJ, Emmett PM, Heaton KW. Intestinal transit time in the population calculated from self-made observations of defecation. J Epidemiol Community Health. 1993;47:331-3.
39. Bouchoucha M, Devroede G, Dorval E, Faye A, Arhan P, Arsac M. Different segmental transit times in patients with irritable bowel syndrome and "normal" colonic transit time: is there a correlation with symptoms? Tech Coloproctol. 2006;10:287-96. 